\title{
Le centre de ressources pédagogiques (CRP) et les nouvelles technologies de l'information et des communications (NTIC) en éducation
}

\section{The Pedagogical Resource Centre (PRC) and New Information and Communication Technologies (NICT) in Education El centro de recursos pedagógicos (CRP) y las nuevas tecnologías de la información y de la comunicación (NTIC) en educación}

\section{Michel Boucher}

Volume 26, numéro 2, automne 1998

Les bibliothèques à l'ère électronique dans le monde de l'éducation

URI : https://id.erudit.org/iderudit/1080637ar

DOI : https://doi.org/10.7202/1080637ar

Aller au sommaire du numéro

Éditeur(s)

Association canadienne d'éducation de langue française

ISSN

0849-1089 (imprimé)

1916-8659 (numérique)

Découvrir la revue

Citer cet article

Boucher, M. (1998). Le centre de ressources pédagogiques (CRP) et les nouvelles technologies de l'information et des communications (NTIC) en éducation. Éducation et francophonie, 26(2), 32-39. https://doi.org/10.7202/1080637ar
Résumé de l'article

La formation des nouvelles technologies de l'information et des communications (NTIC) dans nos facultés d'éducation en milieu minoritaire francophone se fait peu dans les centres de ressources pédagogiques (CRP). Ceux-ci risquent donc de voir leur rôle remis en question, à moins qu'ils ne s'engagent à court terme à devenir d'authentiques lieux d'apprentissage des NTIC, au même titre que toute autre forme de matériel pédagogique.
Tous droits réservés ( $)$ Association canadienne d'éducation de langue française, 1998 services d'Érudit (y compris la reproduction) est assujettie à sa politique d'utilisation que vous pouvez consulter en ligne. 


\section{Le centre de ressources pédagogiques (CRP) et les nouvelles technologies de l'information et des communications (NTIC) en éducation}

\section{Michel BOUCHER}

Université d'Ottawa, Ontario, Canada

\section{RÉSUMÉ}

La formation des nouvelles technologies de l'information et des communications (NTIC) dans nos facultés d'éducation en milieu minoritaire francophone se fait peu dans les centres de ressources pédagogiques (CRP). Ceux-ci risquent donc de voir leur rôle remis en question, à moins qu'ils ne s'engagent à court terme à devenir d'authentiques lieux d'apprentissage des NTIC, au même titre que toute autre forme de matériel pédagogique. 


\section{ABSTRACT}

\section{The Pedagogical Resource Centre (PRC) and New Information and Communication Technologies (NICT) in Education}

Michel BOUCHER

Université d'Ottawa, Ontario, Canada

The development of new information and communication technologies (NICT) is making little headway in the Pedagogical Resource Centres (PRC) of education faculties in areas where francophone students form a minority. These centres risk seeing their role called into question, unless they soon commit themselves to becoming authentic learning centres where NICTs are used alongside any other form of pedagogical material.

\section{RESUMEN}

\section{El centro de recursos pedagógicos (CRP) y las nuevas tecnologías de la información y de la comunicación (NTIC) en educación}

Michel BOUCHER

Université d'Ottawa, Ontario, Canada

La introducción de nuevas tecnologías de la información y de la comunicación (NTCI) en nuestras facultades de educación en medio francófono minoritario se realiza lentamente en los Centros de Recursos Pedagógicos (CRP). Estos corren el riesgo de que su rol se vea cuestionado si no se convierten rápidamente, en auténticos centros de aprendizadje de las NTCI como si fueran una forma más de material pedagógico.

\section{Introduction}

Le sujet de notre propos est de parcourir les centres de ressources pédagogiques (CRP) que fréquente la clientèle universitaire des facultés d'éducation, en particulier dans certains milieux francophones minoritaires du Canada. Constatant la popularité croissante des CD-ROM et d'Internet dans nos centres, nous voulons faire état des CRP comme lieux d'apprentissage pour l'utilisation des NTIC. De plus, nous croyons qu'il en va de la pertinence même des CRP d'inclure dans leur mandat l'expérimentation des NTIC, au même titre que tout autre matériel pédagogique. Nous allons donc vérifier l'état de la situation à la Bibliothèque Saint-Jean de la Faculté Saint-Jean/ 
University of Alberta (Edmonton); celle-ci entre plutôt dans la catégorie des bibliothèques/centres d'information $(\mathrm{BCI})^{1}$ en raison des deux grandes collections qu'elle abrite. Nous allons aussi nous rendre au CRP rattaché à la Bibliothèque Champlain de l'Université de Moncton (Nouveau-Brunswick) et au Centre provincial des ressources pédagogiques (CPRP), situé sur le campus de l'Université Sainte-Anne, à Pointe-de-l'Église (Nouvelle-Écosse). Ce centre est rattaché directement au ministère de l'Éducation pour le prêt aux écoles et à la Faculté d'éducation pour le service à la clientèle universitaire. Enfin, nous terminerons notre parcours au CRP de l'Université d'Ottawa.

\section{Les nouvelles technologies de l'information et des communications (NTIC)}

Un enseignant donne une leçon sur les oiseaux tropicaux du Brésil. Dans une situation virtuellement idyllique, il se branche à Internet dans sa salle de classe et il raccorde son ordinateur portable à un afficheur à cristaux liquides $(L C D)$, permettant ainsi à ses élèves de suivre le déroulement de la séance sur un écran géant. Les interlocuteurs se sont entendus antérieurement sur les protocoles et les contenus de la discussion ainsi que sur les modalités techniques telles que l'utilisation d'un appareil permettant aux interlocuteurs de se voir en communiquant à distance.

Étant donné que l'enseignant est très prévoyant, il enregistrera la séance Internet sur vidéocassette. Ensuite, il numérisera cette information en utilisant un logiciel qui permet de transférer l'information de la vidéocassette dans un fichier ordinolingue. Cette dernière fonction a pour but d'intégrer des sections de la séance Internet ultérieure dans un texte quelconque saisi en mode de traitement de texte ou de traitement d'image. De plus, cet enseignant veut constituer une collection de photographies des oiseaux tropicaux pour ensuite intégrer les photos dans ce même fichier ordinolingue. Il prend donc des photographies avec un appareil photo numérique dont il déverse directement l'information numérisée dans le fichier à l'aide du branchement d'un fil entre l'appareil et l'ordinateur. Finalement, l'enseignant place un magnifique croquis couleur pastel sur le balayeur optique. Il accomplit un petit travail rapide et facile d'édition d'images avec un logiciel de traitement d'images, puis il déverse le document dans le même fichier ordinolingue.

Imaginons, pour revenir à la séance proprement dite, la réaction des élèves en communication directe avec un spécialiste des oiseaux tropicaux au Brésil. Les élèves sont en mode interactif et participent donc directement à l'échange en cours. Ils peuvent saluer l'interlocuteur aussi bien de la voix que du regard. La séance terminée, l'enseignant pourra la reproduire en différé grâce à la vidéocassette. Et si l'équipement mentionné ci-dessus était remis entre les mains des élèves eux-mêmes, nous serions étonnés de la rapidité avec laquelle ils apprennent à manipuler des

1. Une expression que nous empruntons à madame Paulette Bernhard: La vraie nature des bibliothèques scolaires, Documentation et bibliothèques, 40(4), oct.-déc. 1994, p. 197-204. 
appareils. Nous serions également surpris de la qualité de la production qui en résulte, que ce soit un journal de classe fait en collectivité ou un numéro thématique sur les anciennes forteresses de Rome.

Nous voici engagés dans une nouvelle aventure pédagogique, comme l'atteste la mise en scène qui précède. Voilà qu'éclatent nos vieux schèmes architectoniques de temps, de lieu et d'espace. En effet, nous devinons que les NTIC transforment subrepticement et peu à peu la nature des rapports entre les enseignantes ou les enseignants et les élèves dans le geste d'appropriation et de partage des connaissances. Les élèves sont situés dans un espace modulaire défini par le médium. L'enseignante ou l'enseignant, pour sa part, les accompagne au cours de leur navigation dont il a proposé à l'avance le point de destination. Très souvent, nous imaginons que s'édifieront de plus en plus des équipes de navigation avec un capitaine qui ne sera pas nécessairement l'enseignant, mais plutôt quelqu'un choisi parmi les pairs. L'enseignante ou l'enseignant sera plutôt celui qui, du haut du mât, lorgne l'horizon et maintient le cap.

Toute cette activité entamée dans une salle de classe peut être poursuivie et achevée dans le CRP, qui devient ainsi le point de rendez-vous privilégié, en certains cas unique, de l'école en tant que lieu d'apprentissage des NTIC. Le CRP peut aussi constituer le point de départ ou de support logistique de cette expérience réalisée ultérieurement dans la salle de classe. Les habiletés que ces activités requièrent seront idéalement acquises dans le cadre des programmes de formation pédagogique offerts à l'université. À cet égard, les CRP universitaires peuvent participer concrètement à la réalisation de projets pédagogiques utiles et exportables dans le milieu scolaire.

\section{Idées générales sur les NTIC en éducation}

À l'orée du troisième millénaire, nous sommes témoins d'un moment extraordinaire dans l'évolution de l'enseignement et de la pédagogie. Le développement accéléré des nouvelles technologies de l'information et des communications ainsi que le phénomène de la mondialisation de l'information à travers le World-WideWeb ont eu un impact considérable sur l'éducation. Ils ont suscité un éveil, une réflexion approfondie sur le contenu et les méthodes d'enseignement un peu partout. Il nous faut mentionner, sur un plan mondial, le rapport à l'UNESCO de la Commission internationale sur l'éducation pour le vingt et unième siècle, présidée par monsieur Jacques Delors et intitulée L'éducation - Un trésor est caché dedans ${ }^{2}$. Il s'agit d'un plaidoyer pour doter le système éducatif non seulement de maîtres et de professeurs bien formés, mais aussi des outils nécessaires à une éducation de qualité: livres, moyens modernes de communication, environnement culturel et économique de l'école... ${ }^{3}$. Les établissements d'enseignement universitaire sont

2. J. Delors, L'éducation - Un trésor est caché dedans, Paris, Odile Jacob, 1996.

3. Ibid., p. 27. 
prêts à assumer leur rôle quant au premier paradigme et ils laissent au niveau politique, c'est-à-dire aux ministères de l'Éducation des provinces, le soin de prendre en charge le second.

\section{Les CRP pour la diffusion de l'utilisation des NTIC}

Bien que Jacques Delors ne traite pas spécifiquement des bibliothèques/centres d'information dans son rapport L'éducation - Un trésor est caché dedans, il reconnaît que «ces technologies nouvelles sont en train d'engendrer sous nos yeux une véritable révolution qui affecte tout autant les activités liées à la production et au travail que les activités liées à l'éducation et à la formation ${ }^{4}{ }$. Ce dernier point de vue aura donc une incidence significative sur le rôle des CRP comme lieux d'apprentissage des NTIC dans le cadre des facultés d'éducation.

Les CRP desservant une clientèle universitaire francophone en milieu minoritaire varient considérablement d'un endroit à l'autre en ce qui concerne la diffusion de l'utilisation des NTIC. La Bibliothèque Saint-Jean, récemment installée dans ses nouveaux locaux inaugurés en janvier 1996, abrite une collection de ressources pédagogiques et une collection de monographies et de périodiques totalisant la somme de 180000 documents, pour soutenir les besoins de recherche des étudiantes et des étudiants inscrits aux programmes en arts, en éducation et en sciences offerts entièrement en langue française. Le programme en éducation est le plus important des trois et, depuis quelques années, la Faculté Saint-Jean offre également un programme de maîtrise en éducation. Comme le mentionne d'ailleurs la directrice de la bibliothèque, madame Juliette J. Henley, «nous visons à offrir aux futurs enseignants un programme de formation aux nouvelles technologies [...] Nous laissons, cependant, au secteur de l'éducation la tâche de former les étudiants sur l'application de ces technologies dans leur enseignement».

De plus, à l'intérieur de la bibliothèque, il y a une salle d'instruction/laboratoire pourvue de 16 ordinateurs pour les consultations et d'un poste d'instructeur donnant accès au catalogue GATE, aux bases de données disponibles en direct à partir du GATE, de même qu'à Internet. La Bibliothèque Saint-Jean offre une solide formation documentaire intégrant les CD-ROM et Internet. Trois bibliothécaires et plusieurs bibliotechniciennes et bibliotechniciens et commis contribuent à la prestation des services divers de la Bibliothèque Saint-Jean, laquelle a aussi son site Web. De plus, la Faculté Saint-Jean offre le cours CU ME 347 - Utilisation de l'ordinateur en éducation. Ce cours comprend, entre autres, l'initiation aux principales applications de l'ordinateur et Internet comme outil indispensable pour l'apprentissage. Nous constatons ainsi l'existence de liens étroits entre les programmes de la faculté et ceux de la Bibliothèque Saint-Jean sur les plans de la formation documentaire et de la formation aux applications pédagogiques des NTIC et, notamment, quant à la diffusion de l'utilisation des NTIC à partir des CRP en milieu scolaire. Incidemment, il y a eu des

4. Ibid., p. 194. 
ateliers d'élaboration de projets Internet à la Bibliothèque Saint-Jean à l'occasion de l'Institut d'été ACREF-Faculté Saint-Jean, en août $1997^{5}$.

Ce premier institut fut l'occasion, pour les enseignantes et les enseignants francophones des provinces de l'Ouest, y compris les Territoires, de jeter un regard nouveau sur la pédagogie de l'Ouest francophone et de créer des solidarités autour de projets concrets. Ce qui comprenait, entre autres, les réseaux à distance et la création de projets sur Internet. À titre d'exemple, des jumelages entre classes d'élèves francophones ont été réalisés afin que ceux-ci puissent vivre la réalité francophone par des échanges fructueux sur des expériences locales. Ce genre de projet a aussi pour effet de sortir les communautés francophones de l'Ouest de leur isolement. Les ateliers de formation sur Internet ont été bien appréciés et étaient animés par madame Céline Renaud-Charette, du Conseil des écoles publiques de langue française d'Ottawa-Carleton. Les 25 enseignantes et enseignants présents ont pris la décision de se "réseauter" afin de poursuivre la réflexion et les échanges entamés à Edmonton. Voilà donc une belle démonstration d'un CRP comme lieu de diffusion de l'utilisation des NTIC en milieu scolaire.

Le Centre de ressources pédagogiques de l'Université de Moncton est un endroit abritant du matériel documentaire et de curriculums (environ 30000 documents), pour l'utilisation des étudiantes et des étudiants inscrits au programme de formation à l'enseignement. Le bibliothécaire en place, monsieur Léonard Gallant, est la seule personne qui y travaille à temps plein. Le Centre a son site Web CRP. On y mentionne que «le Centre veut favoriser les liens entre le système scolaire et la Faculté des sciences de l'éducation et aussi fournir de la documentation aux chercheuses et aux chercheurs pour qu'ils puissent créer, adapter ou expérimenter du matériel pédagogique».

La description n'est cependant pas explicite sur la question des nouvelles technologies, à part une mention brève de logiciels dans sa collection. Il y a quelques CDROM, de même que l'accès à Internet pour le personnel de la faculté d'éducation, «mais pas pour le public pour l'instant... ça va venir [...] En ce qui regarde le multimédia, ceci relève du secteur T.I.C. mis sur pied l'année dernière à l'Université de Moncton $^{6}{ }$. Le secteur T.I.C. (technologies de l'information et des communications) dont le site Web TIC porte le même nom, est «un amalgame des départements d'audiovisuel, du centre d'informatique et de la téléphonie [...] dont le mandat est d'offrir du matériel et du support. On y offre à l'occasion des petits ateliers, mais rien de niveau formel et académique. Ce département dessert la clientèle de l'Université de Moncton ${ }^{7}$ ». Il y a bien sûr la Bibliothèque Champlain, qui ressemble dans ses grandes lignes à toutes nos grandes bibliothèques universitaires, avec ses services de consultation sur CD-ROM et Internet. Par ailleurs, la Faculté des sciences de l'éducation de l'Université de Moncton offre une gamme de cours en informatique, soit au niveau du baccalauréat, avec le cours ED 3013 - Application pédagogique de l'ordinateur,

5. L'ACREF (Alliance canadienne des responsables et des enseignantes et des enseignants en français langue maternelle) est une association qui a été fondée en 1988.

6. Courrier électronique reçu de Léonard Gallant le 3 décembre 1997.

7. Robert Baudoin, professeur en informatique, de la Faculté d'éducation de l'Université de Moncton. 
soit au niveau supérieur, avec le cours ED 6226 - Application pédagogique de l'ordinateur.

Le Centre provincial de ressources pédagogiques (CPRP) de l'Université SainteAnne comporte des particularités intéressantes. En effet, ce centre offre «une bibliothèque de prêt abritant une collection de 85000 items incluant, entre autres, des logiciels et des CD-ROM éducatifs [...] et permet de rendre l'ensemble de sa collection disponible aux écoles qu'il dessert par le biais d'Internet [...] il offre également un service de publication [...] et un service d'animation [...] dans le cadre des nouveaux programmes en français ${ }^{8} »$. Mais aucune mention explicite des NTIC n'est faite, à part l'utilisation d'Internet dans la bibliothèque de prêt. Depuis l'année universitaire 1996-1997, le département offre le cours PEDA 3843 - Technologie éducative. Ce cours comprend, entre autres, l'acquisition des connaissances par rapport aux nouvelles technologies de l'information et de la communication et des compétences pédagogiques en ce qui concerne l'intégration et l'emploi de ces technologies dans des classes à l'enseignement primaire et secondaire. Le département "a une salle d'informatique combinée à une salle de micro-enseignement qui comprend six (6) ordinateurs Pentium, une imprimante laser et un scanner ». Nous observons donc que les activités pédagogiques sont complémentaires entre le CPRP et le Département des sciences de l'éducation.

Enfin, il y a le Centre de ressources pédagogiques de la Faculté d'éducation de l'Université d'Ottawa, qui a changé son statut de bibliothèque d'éducation et son mandat pour celui actuel de centre de ressources pédagogiques. Cela a pour but de mettre en relief la nature animée et dynamique du centre comme laboratoire d'expérimentation pédagogique. On y vient pour réaliser des activités seul ou en groupe. De plus, le Centre est désormais rattaché à la Faculté d'éducation, bien qu'il soit, moyennant certaines ententes au profit de la clientèle étudiante, branché sur le catalogue du réseau des bibliothèques et sur ses services techniques. Il veut être avant tout un lieu d'apprentissage des NTIC. Dans chacune des aires dégagées du centre, on trouve le catalogue automatisé, des ordinateurs PC et Mac, des sections de la collection générale qui totalisent approximativement 50000 items de nature pédagogique (trousses, jeux, logiciels, CD-ROM, programmes-cadres et curriculum de l'Ontario, bandes dessinées et littérature enfantine et 150 abonnements à des périodiques) ainsi que des cabinets multimédias (incluant le branchement au câble TVO/TFO pour les émissions éducatives et la câblo-éducation, laquelle donne accès à une multitude de postes supplémentaires). Tout cela présente une réalité de travail en mode virtuel autant que réel. Le centre sert ainsi de cadre pour l'enseignement des NTIC à la faculté et certains membres du personnel participent directement à la prestation du cours PED 3717 - C'est la technologie au service de l'enseignement.

Le Centre de ressources pédagogiques (CRP) de l'Université d'Ottawa, qui produit dans les deux langues officielles du pays, est aussi impliqué dans la diffusion de l'utilisation des NTIC dans les conseils scolaires francophones de la région. Le directeur du centre, monsieur François Desjardins, travaille en étroite collaboration

8. Jean-Louis Robichaud, directeur du CPRP de la Faculté d'éducation d'éducation de I'Université Sainte-Anne. 
avec les conseils scolaires pour la réalisation de projets communs comme celui intitulé De la craie à la souris. "Des étudiants-maîtres de l'Université d'Ottawa seront placés dans sept classes différentes du cycle primaire (des Conseils scolaires de langue française du district d'Ottawa) afin d'aider les enseignants. L'accent sera mis sur la production de matériel qui facilitera l'utilisation de la technologie en salle de classe. Une équipe de soutien composée d'enseignants, de partenaires communautaires et du personnel de l'Université d'Ottawa supervisera le projet.»

Ces étudiantes et étudiants en éducation ont été formés au préalable au Centre de ressources pédagogiques.

Autre fait notable, le cours PED 3717 - La technologie au service de l'enseignement est offert pour la première fois au printemps 1998 aux enseignantes et aux enseignants qui veulent développer leurs habiletés dans les applications des NTIC en salle de classe. Le Centre de ressources pédagogiques joue donc un rôle important dans la diffusion de l'utilisation des NTIC dans la région.

\section{Conclusion}

Nous avons constaté que le but des CRP du Nouveau-Brunswick et de la Nouvelle-Écosse est d'assurer à nos futurs enseignantes et enseignants les bénéfices d'une expérimentation du matériel pédagogique. La Faculté d'éducation garde pour sa part le rôle de la formation aux applications pédagogiques des NTIC dans des locaux attenants ou complètement séparés des CRP 9 . Par contre, la Bibliothèque Saint-Jean et le Centre de ressources pédagogiques de la Faculté d'éducation de l'Université d'Ottawa se distinguent par leur intégration complète des NTIC sur les lieux. Dans le cas du Centre, la formation et les exercices se font en partie dans les deux laboratoires Mac et IBM situés physiquement à l'intérieur du Centre et en partie en faisant utilisation des cabinets multimédias placés stratégiquement à divers endroits de la collection. Aussi, nous avons pu constater, nous référant aux exemples de la Bibliothèque Saint-Jean et du Centre de ressources pédagogiques de l'Université d'Ottawa, que la formation aux applications pédagogiques des NTIC et la diffusion de l'utilisation des NTIC vers le milieu scolaire à partir des CRP universitaires illustrent bien la contribution significative que ceux-ci peuvent apporter à leur faculté d'éducation; dans le cas contraire, leur rôle risque sérieusement d'être remis en question.

9. Voir, en préparation, De la bibliothèque au centre de ressources multimédias, Montréal, GRICS, coll. Comment informatiser l'école.

Voir aussi I'ouvrage de M. Souchaud et al., Les technologies de l'information au CDI [centre de documentation et d'information], Paris, Hachette, 1997. 